\title{
A Control Concept of a Compensation Load Cell in Terms of Calibration a Cantilever
}

\author{
O. Dannberg ${ }^{1}$, V. Cherkasova $^{2}$, T. Fröhlich $^{3}$ \\ Institute for Process Measurement and Sensor Technology, Technische Universität IImenau \\ PF 1005 65, 98684 IImenau, Germany \\ 1 oliver.dannberg@tu-ilmenau.de \\ 2 valeriya.cherkasova@tu-ilmenau.de \\ ${ }^{3}$ thomas.froehlich@tu-ilmenau.de
}

\section{Summary:}

The article proposes a concept of an automatic load cell control system with compensation of electromagnetic force for the tasks of precision force measurement. The following tasks were solved: mathematical modeling of mechanical processes in the compensation load cell when touching the cantilever, taking into account mechanical disturbances; identification of parameters and computer simulation of processes; development and simulation of the coil current control loop. The purpose of the control was to find a balance between precise control and reducing the oscillation of the control current.

Keywords: control system, load cell, electromagnetic force compensation, cantilever, bumpless control transfer

\section{Introduction}

There are many studies going on today and analyzes of scanning atomic force microscope (AFM). One of the important areas of research for improving the quality of the atomic force microscope is the calibration of the stiffness of the cantilever springs of the device [1], [2]. A force displacement measurement device for the determination of spring constants was developed at TU IImenau [3]. The research was continued in this subject [4]. In these developments the spring constant of AFM cantilevers is determined by using an electromagnetic force compensated (EMFC) load cell, for which analysis and control methods given in the article [5]. However, in the cited works, the concepts of control systems are not considered when changing the overall stiffness coefficient of the system.

The functional principle of the device and mathematical model of mechanical processes in the single joint load cell

The basic principle of measuring the cantilever force is based on a linear relationship between it and the compensation current of coil in the permanent magnet field attached to the weight beam. When touched by a cantilever, the balance beam deviates from the zero position, the deviation is fixed by an interferometer and transmitted to the controller, which in turn changes the current in the coil, thereby the Lorentz force returns the beam to zero.
The main research problem is the development of precise control of the coil current when the cantilever and the weighing beam are in contact. The stages of the position and impact of the cantilever are shown in Fig. 1 (I-III).

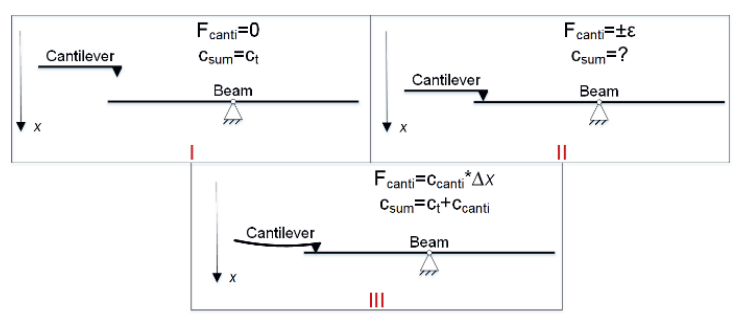

Fig. 1. I. The cantilever does not touch the beam. II. The moment of the contact of the cantilever and the beam. III. The cantilever touches the beam

In this figure: $c_{\text {sum }}$ - the overall stiffness coefficient; $c_{t}$ - the flexure hinge spring constant; $c_{\text {canti }}$ - the cantilever stiffness coefficient.

A simplified representation of the device is shown in Fig. 2.

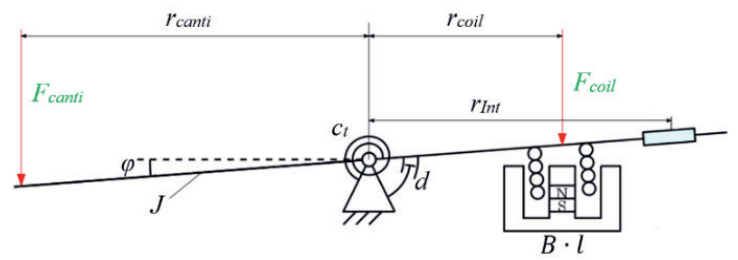

Fig. 2. Simplified mechanical representation of the beam 
In this scheme: $J-$ moment of inertia of the beam; $d$ - the damping coefficient of the damper between flexure hinge and beam; $\varphi-$ the beam angular displacement; $r_{I n t}-$ the distance between mirror of the interferometer and joint. Based on the balance moments of forces a system of differential equations is derived (1).

$$
\left\{\begin{array}{l}
\dot{q}_{1}=q_{2} \\
\dot{q}_{2}=-\frac{c_{t}+c_{\text {canti }} r_{\text {canti }}{ }^{2}}{J} q_{1}-\frac{d}{J} q_{2}+\frac{M_{\text {canti }}}{J}-\frac{M_{\text {coil }}}{J}
\end{array}\right.
$$

Computer model is performed in the Matlab / Simulink software.

\section{Control system}

As can be seen from Fig. 1, at the moment of cantilever contact with the weighing beam, the impact force and the total system stiffness coefficient are unknown. The dynamic behavior of the system changes suddenly. The main objective of the control is to improve the balancing of the beam at the touch of the cantilever, reduce the transient time and reduce the current oscillations. It is proposed to use bumpless control transfer between two discrete PID controllers. If the error modulus between the set position and the position signal from the interferometer is greater than selected error value, the fast controller is triggered, which adjusts the deviation faster, if the error modulus is less than selected error value, the slow controller is triggered.

Since the system error varies greatly when the cantilever comes in contact with the balance beam, the control system must take into account the moment the cantilever is touched and change the error value on the switch, an observer system is needed. The integral of the error squared between the signals from the object and the observer allows you to take into account additional noise at the object, which does not affect the observer in real time. The full structure of the control system consists from four PID controllers and three switches. The control frequency is $600 \mathrm{~Hz}$.

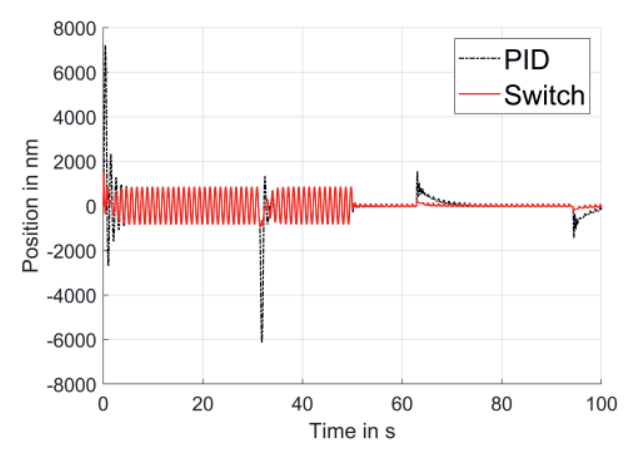

Fig. 3. Comparing simulated displacements of the beam balance with control

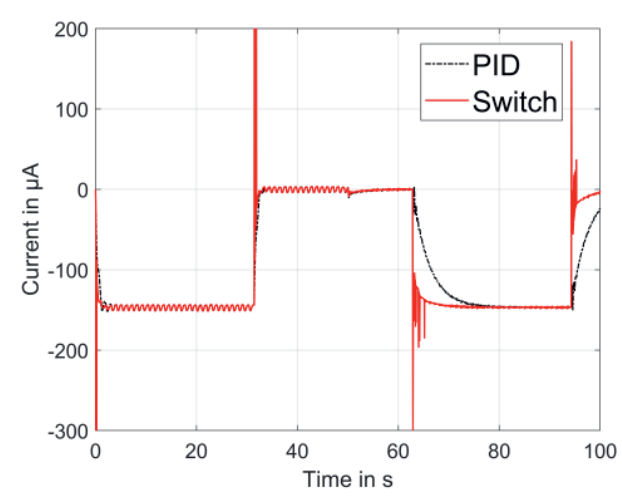

Fig. 4. Comparing simulated currents

Figures 3 and 4 show control results when comparing the developed control system and the slow PID controller. The response rate of the system at contact of the EMFC load cell with the cantilever is improved by 8.39 seconds, thus reducing the time of the transition process. Overshoot reduced by $72 \%$. The control current oscillations are acceptable.

This work was supported by the Deutsche Forschungsgemeinschaft (DFG) in the scope of the Research Training Group "Tip- and Laserbased 3D-Nanofabrication in extended macroscopic working areas" (GRK 2182) at Technische Universität IImenau, Germany.

\section{References}

[1] J. R. Pratt et al., "Review of si traceable force metrology for instrumented indentation and atomic force microscopy," Measurement science and technology, vol. 16 , no. 11 , p. 2129, 2005; doi:10.1088/0957-0233/16/11/002.

[2] Zhao, Yu Wen, et al. "Accurate and traceable calibration of the stiffness of various AFM cantilevers." Key Engineering Materials. Vol. 645. Trans Tech Publications, 2015; doi:10.4028/www.scientific.net/KEM.645646.817 .

[3] C. Diethold, M. Kühnel and T. Fröhlich (2014). Development of a Force Displacement Measurement Device for the Determination of Spring Constants. Universitätsbibliothek IImenau; urn: urn:nbn:de:gbv:ilm1-2014iwk-092:6.

[4] O. Dannberg, M. Kühnel and T. Fröhlich (2018, July). Nanonewton Force Measurements Based on Electromagnetic Force Compensated Balances (EMFC). In 2018 Conference on Precision Electromagnetic Measurements (CPEM 2018) (pp. 1-2). IEEE; doi: 10.1109/CPEM.2018.8501174.

[5] A. Amthor, I. Kaiser, N. Rogge and H. Weiss (2014). Analysis, physically motivated modeling and system identification of electromagnetic force compensated balances (EMFC). In Shaping the Future by Engineering: Proceedings; 58th IWK, IImenau Scientific Colloquium, Technische Universität Ilmenau, 8-12 September 2014 (Vol. 58); urn: urn:nbn:gbv:ilm1-2014iwk:3. 